\title{
A GENERAL HOFFMAN-WERMER THEOREM FOR ALGE BRAS OF OPERATOR FIELDS
}

\author{
D. C. TAYLOR ${ }^{1}$
}

ABSTRACT. Let $A$ be a closed separating subalgebra of $C(T)$ that contains the identity. It is known that $\operatorname{Re} A$ is uniformly closed only if $A=C(T)$. In this note it is shown that this property characterizes all maximal full algebras of operator fields and not just $C(T)$.

1. Introduction. Let $T$ be a compact Hausdorff space and $C(T)$ the space of all complex-valued continuous functions on $T$. It is of interest and importance to know which properties of $C(T)$ cannot be shared with any of its proper separating subalgebras. In other words, which properties of $C(T)$ characterize it. A classic example of such a property, due to Hoffman and Wermer [3], is the following: If $A$ is a closed separating subalgebra of $C(T)$ that contains the identity, then $\operatorname{Re} A$ is uniformly closed only if $A=C(T)$. It is the purpose of this note to extend the Hoffman-Wermer theorem to the setting of a maximal full algebra of operator fields. Thus, it shows that the Hoffman-Wermer property characterizes all maximal full algebras of operator fields and not just $C(T)$.

We will now define a maximal full algebra of operator fields on $T$. For each $t \in T$, let $C_{t}$ be a $C^{*}$-algebra with identity $I_{t^{*}}$ For different values of $t$ the $C_{t}$ are in general unrelated. An operator field (with respect to $\left\{C_{t}\right\}_{t \in T}$ ) is a function $x$ on $T$ such that $x(t) \in C_{t}$ for each $t \in T$. By a full algebra of operator fields on $T$, we mean a set $\mathrm{C}(T)$ of operator fields $x$ on $T$ that satisfy the following: (i) $\mathrm{C}(T)$ is a *-algebra under pointwise algebraic operations; (ii) for each function $x$ in $\mathrm{C}(T)$, the function $t \rightarrow\|x(t)\|$ is continuous; (iii) for each $t \in T,\{x(t): x \in \mathrm{C}(T)\}$ is dense in $C_{t}$; (iv) $\mathrm{C}(T)$ is complete in the norm $\|x\|=\sup \{\|x(t)\|: t \in T\}$. Clearly, $\mathrm{C}(T)$ is a $C^{*}$-algebra; hence (iii) could be strengthened to the statement that $\{x(t): x \in \mathrm{C}(T)\}=C_{t}$. The algebra $\mathrm{C}_{t}$ will be called the component of $\mathrm{C}(T)$ at $t$. An operator field $x$ is continuous at $t_{0}$ (with respect to $C(T)$ ) if for each $\epsilon>0$ there is an element $y$ of $\mathrm{C}(T)$ and a neighborhood $U$ of $t_{0}$ such that $\|x(t)-y(t)\|<\epsilon$ for all $t \in U$. We say that $x$ is continuous on $T$ if it is continuous at each point of $T$. It is well known that $\mathrm{C}(T)$ is a maximal full algebra of operator fields

Received by the editors March 19, 1974 and, in revised form, August 8, 1974 . AMS (MOS) subject classifications (1970). Primary 46L15, 46L05; Secondary $46 \mathrm{~J} 10$.

1 This research was supported in part by the National Science Foundation, under contract No. GP-38884. 
if and only if it contains all operator fields $x$ which are continuous on $T$ with respect to $\mathrm{C}(T)$. For basic concepts and results on maximal full algebras of operator fields, we refer the reader to [1, Chapter 10] or [2].

2. The general Hoffman-Wermer theorem. Throughout this section $\mathrm{C}(T)$ will denote a maximal full algebra of operator fields. We shall assume that the operator field $t \rightarrow I_{t}$ belongs to $\mathrm{C}(T)$, which we will denote by $I$. Let $A$ be a subalgebra of $\mathrm{C}(T)$. Each $x \in A$ has a unique decomposition $x=h_{1}+$ $i h_{2}$, where $h_{1}$ and $h_{2}$ are hermitian elements of $\mathrm{C}(T)$. We will let $\operatorname{Re} x$ denote $h_{1}$ and $\operatorname{Im} x$ denote $h_{2}$. We will let $\operatorname{Re} A=\{\operatorname{Re} x: x \in A\}$ and $A^{b}=$ $\{x \in A: x$ hermitian $\}$. Note that $\operatorname{Re} A$ and $A^{b}$ are linear subspaces of $A$. Moreover, when $A$ is uniformly closed, so is $A^{b}$. For $x \in A$ we denote by $\sigma(x)$ and $\sigma_{A}(x)$ the spectrum of $x$ with respect to $\mathrm{C}(T)$ and $A$, respectively. Similarly, we define $\sigma_{C_{t}}(x(t))$ for each $t \in T$. If $\xi$ is a normal element in $C_{t}$ and $f$ is a complex-valued continuous function on $\sigma_{C_{t}}(\xi)$, then we let $f(\xi)$ denote the element in $C_{t}$ defined in the usual way for $C^{*}$-algebras.

2.1. Lemma. Let $A$ be a uniformly closed subalgebra of $\mathrm{C}(T)$ and $x$ an element of $A$. Then the following statements are true:

(1) if $x \in A^{b}$ and $f$ is a real-valued continuous function on $\sigma(x) \cup\{0\}$ with $f(0)=0$, then the operator field $t \rightarrow f(x(t))$, denoted $f(x)$, belongs to $A^{h}$;

(2) if $x$ is normal and $f$ is an analytic function in some simply connected region containing $\sigma(x) \cup\{0\}$ with $f(0)=0$, then the operator field $t \rightarrow f(x(t))$ denoted $f(x)$, belongs to $A$.

Proof. The proof follows immediately from the fact that $f$ in both cases ${ }^{4}$ can be uniformly approximated by polynomials of the form $a_{1} \lambda+a_{2} \lambda^{2}+\cdots+a_{n} \lambda^{n}$.

2.2. Definition. A subalgebra $A$ of $\mathrm{C}(T)$ is said to satisfy the $\mathrm{H}-\mathbb{W}$ condition if the following statements hold: (1) $\operatorname{Re} A$ is uniformly closed; (2) for each nonsingleton compact subset $F$ of $T$ and $t_{0} \in F$ there is a $t_{1} \in F$ and a normal element $x$ in $A$ such that $x\left(t_{0}\right)=0, x\left(t_{1}\right)=I_{t}$, and $\sup \{\|x(t)\|: t \in F\}$ $=1$.

Note that in the function algebra setting of Hoffman and Wermer the algebra $A$ separates points of $T$ if and only if condition (2) of 2.2 holds. Consequently, the following result extends the Hoffman-Wermer theorem to the setting of a maximal full algebra of operator fields. Furthermore, it extends [1, 10.4 .6$, p. 197] and partially extends $[1,11.5 .3$, p. 234] to subalgebras of $\mathrm{C}(T)$ that are not necessarily selfadjoint.

2.3. Theorem (Hoffman-Wermer). Suppose $A$ is a uniformly closed subalgebra of $\mathrm{C}(T)$ that satisfies the $H-W$ condition. Then for each complexvalued continuous function $f$ on $T$ the operator field $t \rightarrow f(t) I_{t}$ belongs to $A$. Moreover, if $A_{t}=C_{t}$ for each $t \in T$, then $A=\mathrm{C}(T)$. 
Proof. Let $t_{0} \in T$ and $U$ the set of all $t \in T$ for which there is an $x$ in $A^{h}$ such that $x(t)=I_{t}$ and $x\left(t_{0}\right)=0$. Suppose there exists a $t_{1} \in T$ and an $x \in A^{b}$ such that $\sigma_{C_{t_{0}}}\left(x\left(t_{0}\right)\right) \cup\{0\}$ and $\sigma_{C_{t_{1}}}\left(x\left(t_{1}\right)\right)$ are disjoint. Let $V_{0}$ and $V_{1}$ be open neighborhoods of $\sigma_{C_{t_{0}}}\left(x\left(t_{0}\right)\right) \cup\{0\}$ and $\sigma_{C_{t_{1}}}\left(x\left(t_{1}\right)\right)$, respectively, with disjoint closures. By $[1,10.3 .6, \mathrm{p} .195]$ there exist open neighborhoods $W_{0}$ and $W_{1}$ of $t_{0}$ and $t_{1}$, respectively, such that $\sigma_{C_{t}}(x(t)) \subseteq V_{0}$ for all $t \epsilon$ $W_{0}$ and $\sigma_{C_{t}}(x(t)) \subseteq V_{1}$ for all $t \in W_{1}$. By Urysohn's lemma there is a realvalued continuous function $p$ such that $0 \leq p(\lambda) \leq 1$ for all real $\lambda, p(\lambda)=0$ for all $\lambda$ in $V_{0}$, and $p(\lambda)=1$ for all $\lambda$ in $V_{1}$.

By 2.1, $p(x) \in A$ and it follows that $p(x)(t)=p(x(t))=0$ for $t \in W_{0}$ and $p(x)(t)=p(x(t))=I_{t}$ for $t \in W_{1}$. Hence $U$ is open and equal to the set of all $t \in T$ for which there is an $x$ in $A^{b}$ such that $\sigma_{C_{t}}(x(t))$ and $\sigma_{C_{t_{0}}}\left(x\left(t_{0}\right)\right) \cup$ $\{0\}$ are disjoint. Actually, our argument tells us more. Let $K$ be a compact subset of $U$. By adapting our argument we can show that there is a positive element $x$ in the unit ball of $A$ and a neighborhood $V$ of $t_{0}$ such that $x(t)=$ $I_{t}$ for $t \in K$ and $x(t)=0$ for $t \in V$.

Now let $F=T \backslash U$. Suppose $F \neq\left\{t_{0}\right\}$. By virtue of the $\mathrm{H}-\mathbb{W}$ condition there is a $t_{1} \in F$ and a normal element $y$ in $A$ such that $y\left(t_{0}\right)=0, y\left(t_{1}\right)=$ $I_{t_{1}}$, and $\sup \{\|y(t)\|: t \in F\}=1$. Since $\operatorname{Re} A$ is uniformly closed, we have, by virtue of the open mapping theorem, a positive integer $n$ for which the following statement holds: if $u \in \operatorname{Re} A$ with $\|u\|<1$, then there exists an $x \in$ $A$ such that $\operatorname{Re} x=u$ and $\|x\|<n$. Let

$$
\eta=[\exp (2 n+1) \pi+1] /[\exp (2 n+1) \pi-1],
$$

and let $D_{\eta}$ and $R$ be the regions in the complex plane defined by $D_{\eta}=\{\lambda$ : $|\lambda|<\eta\}$ and $R=\{\lambda:-1 / 2<\operatorname{Im} \lambda<1 / 2\}$. By using simple combinations of wellknown conformal maps it is easy to deduce that the function $g$ defined on $D_{\eta}$ by the formula

$$
g(\lambda)=[1 / \pi][\ln (1+\lambda / \eta)-\ln (1-\lambda / \eta)]
$$

maps $D_{\eta}$ onto $R$. Moreover, $g(0)=0, g(1)=2 n+1$, and the Taylor series expansion of $g$ is given by

$$
g(\lambda)=[2 / \pi] \sum_{k=0}^{\infty}[1 /(2 k+1)][\lambda / \eta]^{2 k+1} .
$$

Since the coefficients of the Taylor series expansion are real and the convergence is uniform on $\bar{D}_{1}=\{\lambda:|\lambda| \leq 1\}$, there exists a positive integer $m$ and a positive number $\alpha$ such that the function $h$ defined on $\bar{D}_{\lambda}$ by the formula

$$
h(\lambda)=[2 \alpha / \pi] \sum_{k=0}^{m}[1 /(2 k+1)][\lambda / \eta]^{2 k+1}
$$


has the following properties: $h(0)=0 ; h(1)=2 n+1 ;-1<\operatorname{Im} h(\lambda)<1$ for all $\lambda \in \bar{D}_{1}$. Let $K=\{t \in T:\|\operatorname{Im} h(y)(t)\| \geq 1\}$. Since $y$ is normal, it follows from 2.1 that $K$ is a compact subset of $U$. Therefore there is a positive $z$ in the unit ball of $A$ such that $z(t)=I_{t}$ for $t \in K$ and $z\left(t_{0}\right)=0$. Set

$$
w_{1}=(I-z)(h(y))(I-z)+(2 n+1)\left(2 z-z^{2}\right)
$$

It is easy to verify that $w_{1}\left(t_{0}\right)=0, w_{1}\left(t_{1}\right)=(2 n+1) I_{t_{1}}$, and $\left\|\operatorname{Im} w_{1}\right\|<1$. As remarked earlier, we have by the open mapping theorem an element $w_{2}$ in $A$ such that $\operatorname{Re} w_{2}=\operatorname{Re} i w_{1}$ and $\left\|w_{2}\right\| \leq n$. Set $x=w_{1}+i w_{2}$, which clearly belong to $A^{b}$. Since

$$
\sigma_{C_{t_{1}}}\left(x\left(t_{1}\right)\right)=2 n+1+\sigma_{C_{t_{1}}}\left(i w_{2}\left(t_{1}\right)\right) \subseteq[n+1,3 n+1]
$$

and

$$
\sigma_{C_{t_{0}}}\left(x\left(t_{0}\right)\right)=\sigma_{C_{t_{0}}}\left(i w\left(t_{0}\right)\right) \subseteq[-n, n],
$$

it follows that $t_{1} \in U$, which is a contradiction. Hence $T \backslash U=\left\{t_{0}\right\}$.

Let $K_{1}$ and $K_{2}$ be pairwise disjoint compact subsets of $T$. Since $t_{0}$ was chosen arbitrarily, it is straightforward to show there exists a positive element $x$ in the unit ball of $A$ such that $x(t)=I_{t}$ for $t \in K_{1}$ and $x(t)=0$ for $t \in K_{2}$. Now let $f$ be a complex-valued continuous function on $T$. We wish to show that the operator field $t \rightarrow f(t) I_{t}$ belongs to $A$. Clearly, we may assume $f$ is positive and $\|f\|_{\infty}=1$. Let $\epsilon>0$.

Choose a positive integer $n$ so that $5 / n<\epsilon$. For each integer $j, 1 \leq j$ $\leq n$, set

$$
F_{j}=\{t \in T:(j-1) / n \leq f(t) \leq j / n\}
$$

It is clear that $\left\{F_{j}\right\}_{j=1}^{n}$ is a collection of compact sets such that $\bigcup_{j=1}^{n} F_{j}=T$. Now for each $j$ define the open set

$$
V_{j}=\{t \in T:(2 j-3) / 2 n<f(t)<(2 j+1) / 2 n\}
$$

which clearly contains $F_{j}$. We now know there are positive elements $\left\{z_{j}\right\}$ in the unit ball of $A$ such that $z_{j}(t)=I_{t}$ for $t \in F_{j}$ and $z_{j}(t)=0$ for $t \in T \backslash V V_{j}$. Since each $t \in T$ belongs to some $F_{j}, z(t) \geq z_{j}(t)=I_{t}$, where $z=\sum_{k=1}^{n} z_{k}$. Thus $\sigma_{C_{t}}(z(t)) \geq 1$ for all $t \in T$. By virtue of 2.1 it is easy to see that the operator field $t \rightarrow z(t)^{-1}$ belongs to $A$. Set $x_{j}=z^{-1 / 2} z_{j} z^{-1 / 2}$ for $j=1,2, \ldots$, $n$. Let $t \in T$. Then there is an $F_{j}$ such that $t \in F_{j}$. Moreover, the element $t$ must belong to $V_{j}$ and possibly $V_{j-1}$ or $V_{j+1}$, but no other open set from our collection $\left\{V_{k}\right\}_{k=1}^{n}$. Note that $\sum_{k=1}^{n} x_{k}=I$, so 


$$
\begin{aligned}
\left\|f(t) I_{t}-\sum_{k=1}^{n} \frac{k-1}{n} x_{k}(t)\right\| & =\left\|\sum_{k=1}^{n}\left[f(t)-\frac{k-1}{n}\right] x_{k}(t)\right\| \\
& \leq \sum_{k=j-1}^{j+1}\left|f(t)-\frac{k-1}{n}\right| \leq \frac{5}{n}<\epsilon .
\end{aligned}
$$

Thus the operator field $t \rightarrow f(t) I_{t}$ belongs to $A$ since $A$ is uniformly closed.

Finally we wish to show $A=\mathrm{C}(T)$ if $A_{t}=C_{t}$ for each $t \in T$. Note that $A$ separates points of $T$ in the sense of [4, p. 177] and that each closed subset of $T$ is an intersection of peak sets in the sense of [4, Definition 4.5, p. 181]. So by [4, Theorem 4.6, p. 182], [4, Corollary 4.2, p. 179], and [4, The orem 4.15, p. 186], $A=\mathrm{C}(T)$.

\section{REFERENCES}

1. J. Dixmier, Les $C^{*}$-algèbres et leurs représentations, Cahiers Scientifiques, fasc. 29, Gauthier-Villars, Paris, 1969. MR 39 \#7442.

2. J. Fell, The structure of algebras of operator fields, Acta Math. 106 (1961), 233-280. MR $29 \# 1547$.

3. K. Hoffman and J. Wermer, A characterization of $C(X)$, Pacific J. Math. 12 (1962), 941-944. MR $27 \# 325$.

4. D. Taylor, Interpolation in algebras of operator fields, J. Functional Analysis 10 (1972), 159-190.

DEPARTMENT OF MATHEMATICS, MONTANA STATE UNIVERSITY, BOZEMAN, MONTANA 59715 